\title{
Periodontal disease and its impact on general health in Latin America. Section IV: Diagnosis
}

\section{Cassiano Kuchenbecker RÖSING(a,b) \\ Juliano CAVAGNI(a,b) \\ Zilson MALHEIROS(b,c) \\ Bernal STEWART ${ }^{(b, c)}$ (D) \\ Vicente ARÁNGUIZ FREYHOFER ${ }^{(\mathrm{d})}$}

(a) Universidade Federal do Rio Grande do Sul - UFRGS, Faculty of Dentistry, Department of Periodontology, Porto Alegre, RS, Brazil.

(b)Latin American Oral Health Association LAOHA, São Paulo, SP, Brazil.

(c) Colgate Palmolive Company, Global Technology Center, Piscataway, NJ, USA.

(d) Universidad de los Andes, Facultad de Odontología, Department of Cariology, Santiago, Chile.

Declaration of Interests: The authors certify that they have no commercial or associative interest that represents a conflict of interest in connection with the manuscript.

\section{Corresponding Author:}

Cassiano Kuchenbecker Rösing

E-mail: ckrosing@hotmail.com

https://doi.org/10.1590/1807-3107bor-2020.vol34.0022

Submitted: September 2, 2019

Accepted for publication: September 22, 2019

Last revision: September 29, 2019
Abstract: The art of diagnosis is of great importance in the management of any disease. This includes preventive and therapeutic strategies. To make an accurate and effective diagnosis, knowledge about the health-disease process is fundamental. This paper reviews the important aspects for periodontal diagnosis in a contemporary approach, and endeavors to establish challenges for improving periodontal diagnosis, especially in Latin America. Considering that contemporary periodontal diagnosis should be based on knowledge of the etiopathogenesis of periodontal diseases, this paper highlights that the recently proposed classification system for periodontal diseases and conditions was based on the best available evidence. This system was conceived for individual diagnosis, therefore, its use in research and epidemiological settings might be limited. The system leads to a practical implication that stresses the importance of interviewing the patient, thorough periodontal charting, and requesting any imaging and other complementary tests necessary. An important observation is that partial periodontal data recordings usable for screening are not diagnostic methods and might underestimate disease. The goals of utmost importance for Latin America are to increase the awareness of both the population and the profession and to prioritize correct periodontal diagnosis. In addition, learning how to use the new classification system will help with diagnosing periodontal diseases.

Keywords: Diagnosis; Periodontal Diseases; Latin America.

\section{Introduction}

Diagnosis of periodontal diseases and conditions has been subject to a series of controversies that led to difficulties in communication, and especially to different clinical approaches in Dentistry. The art of diagnosis should be considered above any classification system which, per se, is an arbitrary way of distinguishing different forms of disease and conditions. In this sense, this paper will make a contemporary approach to the diagnosis of the periodontal health/disease process, in an endeavor to understand its challenges, and to propose possible solutions, especially for Latin American countries. The paper was part of a Workshop including experts from Latin America. Contributions from the discussion are included in the article. 
Historically, Dentistry has focused mainly on dental caries, since this was the major cause of tooth loss, pain, and impairment of oral health. This has led to a practice of underdiagnosis of periodontal diseases and conditions. After the decline in the occurrence of dental caries, and advent of understanding the importance of more comprehensive oral care, periodontal diseases need to be looked upon with more attention, from a health perspective of individuals and populations. This is supported by evidence of the role of periodontal diseases in oral outcomes e.g. tooth loss, as well as possible relations with other systemic conditions and oral health-related quality of life..$^{1,2,3}$ Studies have demonstrated that routine periodontal diagnosis is not performed as it would be expected. Several clinicians even have trouble with making a diagnosis, or they might find more than one differential diagnosis. ${ }^{2,4}$ This is probably a reflection of how periodontal diagnosis is approached in dental schools and the way the health systems value periodontal clinical assessment and management.

\section{Diagnosis of the periodontal health/disease process}

One important aspect that surrounds the art of diagnosis of periodontal health/disease process is the distinction between epidemiological and clinical settings. The aim of epidemiological surveys is to describe the occurrence of health and disease states in populations, associating them with possible risk factors/indicators. Therefore, epidemiological studies are not focused on individual diagnosis as such. The misunderstanding of the role of these epidemiological surveys has led to confusion in terms of diagnosis of periodontal diseases. Diagnosis of periodontal diseases from an individual perspective should be focused on the person as a whole. In this sense, data from epidemiological studies will be used to build the knowledge that will guide the process of diagnosis. There is one point of consensus: periodontal disease cannot be diagnosed after the tooth is about to be lost due to periodontal breakdown.

Periodontal diseases have been classified in different ways. The point that needs to be reinforced is that the periodontal health/disease process clinically manifests in two main types of impairment: gingivitis and periodontitis. The distinction between these two diseases is mainly based on the concomitant occurrence of loss of attachment. Gingivitis is an inflammatory process triggered by the presence of supragingival biofilm, and is not associated with periodontal breakdown. Periodontitis occurs after imbalance between the presence of subgingival biofilm and the host response, leading to loss of periodontal attachment and bone. Since both diseases have a background of an inflammatory process, diagnosis should include these aspects in the interview with the patient, in the physical examination, and with additional diagnostic tests that could help in the diagnosis. ${ }^{5}$

In 2018, a Joint Workshop hosted by both the European Federation of Periodontology and the American Academy of Periodontology launched a new classification system for periodontal and periimplant diseases and conditions. An impressive effort was made to improve the existing classification systems. ${ }^{6}$ Professionals usually require a learning curve to enable the new classification system to be adopted worldwide. The system comprises gingival health, gingivitis, periodontitis and peri-implant diseases and conditions. An in-depth study by the profession is necessary to enable implementation of this new system. The main difference from the existing classification, which was launched by the American Academy of Periodontology in $1999^{5}$, is the fact that a more comprehensive diagnosis process is required when trying to establish case definitions. The system is based on the best available evidence, however, in some situations low-level evidence had to be used. An extremely important aspect to understand is that the classification system was not meant to be a priority for epidemiology or research, but was meant for individual diagnosis. Of course, it needs to be understood that the extensive study conducted in the literature should be the basis for epidemiological and research, without the need for complete standardization between these two activities. This paper acknowledges that a part of the new system was dedicated to defining gingival health - from pristine gingival health to clinically 
healthy gingiva. In addition, this paper points out that periodontitis was mainly classified into stages and grades. This system allows the understanding that in each patient, both rate of progression and the way the function is affected, and accounts for tooth loss, for example. The system needs further evaluation, and when considered necessary, improvement. Close attentive reading of the special issues of both Journal of Clinical Periodontology and Journal of Periodontology is strongly recommended, in which the system is thoroughly presented.

\section{Periodontal diagnosis in practice}

This paper emphasizes the importance of general practicioners and specialists being well trained in diagnostic capabilities. Specialists should also perform more in depth examination of complex cases. Furthermore, although this article has focused on periodontal diagnosis, it is mandatory that dentists should be proficient in oral health diagnosis. For example, root caries is a very frequent situation in periodontal individuals, and it should not be underdiagnosed.

The interview with the patient is of utmost importance in periodontal diagnosis. This is a challenge to dental schools, since a comprehensive interview is one of the keys of periodontal diagnosis. For example, in terms of other systemic conditions, classification systems have listed over 50 conditions related to the occurrence of periodontal diseases, from hormonal changes, exposure to environmental factors to rare syndromes. ${ }^{5}$ Professionals need to have this knowledge and incorporate it into the interview with the patient. Moreover, since periodontal diseases are linked to behavioral components including oral hygiene methods, these should be part of the diagnostic process

The physical examination should consider the understanding that periodontal diseases are of a chronic nature. The progression of untreated periodontitis is known to be slow, therefore limiting the rapid clinical impact. ${ }^{7}$ In this sense, periodontal physical examination still is based on the history of disease. Therefore, the most used diagnostic tool is periodontal probing, for the purpose of understanding both the inflammatory status (e.g. with probing depth or bleeding on probing) or the history of disease (with loss of attachment). This is also one of the best tools for monitoring progression of disease over time. ${ }^{8}$ Considering the foregoing information, it is a consensus that in some way, every dentist needs to perform periodontal probing in every patient. The new classification system calls for probing attachment loss to enable better diagnosis of periodontitis. ${ }^{6}$ Of course, for epidemiological reasons, periodontal probing to obtain the history of disease progression should be performed mainly in adult individuals. Children should be periodontally diagnosed with probing and/or radiographs if they have family history of periodontal disease.

Periodontal probing is known to be time consuming and laborious and this is one of the reasons why it has not been as widely used as expected. This paper urges that dental training reinforces the importance of using this tool to increase the quality of oral diagnosis. ${ }^{6}$ Reported differences have been observed for different types of probes (manual vs. automatized/computerized). ${ }^{8}$ Therefore, practitioners are encouraged to use any type of probe. The gold standard for periodontal diagnosis is full mouth periodontal examination, i.e. periodontal probing in six sites per tooth (disto-buccal, mid-buccal, mesio-buccal, disto-palatal/lingual, mid-palatal/ lingual, mesio-palatal/lingual). When this approach is adopted, there are few chances of misdiagnosis of periodontal diseases., ${ }^{910}$ However, other simplified approaches have been proposed in an attempt to increase the number of dentists routinely performing periodontal examinations.

It should be kept in mind that screening is the main aim of any type of partial examination, thus if periodontal disease is found by means of this approach, complete periodontal charting is mandatory. Susin et al. ${ }^{11}$ tested 7 partial recording protocols based both on full mouth and in half mouth examinations and observed that all partial examination protocols underestimated the occurrence of periodontal disease. The best partial recording protocol found in this study was probing 3 sites per tooth (mesio-buccal, mid-buccal and disto-lingual). Nevertheless, this is still time consuming. The more severe the disease is, 
the worse partial recording for periodontal diagnosis will be. Therefore, the recommendation is that if an individual has periodontitis, full-mouth periodontal probing must be performed.

An alternative has been proposed for periodontal screening; that is the so called basic periodontal examination (periodontal screening and recording). ${ }^{4}$ This examination is based on probing all the teeth and scoring the sextant according to probing depth. When deeper probing depths are observed, a full-mouth periodontal examination is recommended. This is an interesting alternative, for those who understand that periodontal charting is not necessary. However, it must be borne in mind that this type of periodontal diagnosis has the potential to underestimate the disease. On the other hand, if this were the only alternative considered for periodontal diagnosis, this would allow screening of more severe cases. Screening is an effective way of covering a larger number of the population. In different settings, this should be subject to discussion. The premise is that "doing something is better than doing nothing". This is partially true, especially in individual situations. It should be emphasized that screening is not diagnosis.

In addition to interviewing the patient and periodontal physical examination, additional diagnostic tests are available. Image tests are the most used in terms of periodontal diagnosis. However, considering the international guidelines for radioprotection, they should be preceded by clinical indication, i.e. data from either the interview with the patient or from the physical examination are the core factors for indicating imaging examinations. The most common image tests used in periodontal diagnosis are periapical and panoramic radiographs, and more contemporarily, the cone-beam computed tomography (CBCT). All of them expose the individual to $\mathrm{x}$-rays and therefore need to be limited. This paper recommends that the practitioners must be aware of the international guidelines for radioprotection before indicating such examinations. ${ }^{12}$

The panoramic radiograph is one of the most costeffective images, however, in cases of periodontal breakdown, it offers limited image detail. Therefore, in cases of moderate disease, complementation with selected periapical or vertical bitewings is warranted, and in cases of severe periodontal disease, a complete periapical radiographic examination could be necessary. The use of CBCT is restricted to specific periodontal situations, including endo-perio relationships, fractures, perforations, etc. ${ }^{13}$

The most important aspect of image tests is that they are comprehensively analyzed, in order to yield a better diagnosis. In the specific case of periodontal diseases, the bone crest deserves special attention, both in terms of the presence of lamina dura (which might be indication of periodontal stability), and the amount of lost periodontal bone, especially for future analyses of disease progression.

Sophisticated diagnostic methods have been proposed in the literature, including microbiological, immunological, physical, molecular assays. ${ }^{14}$ These methods have been extensively used in research. However, for the clinical approach, they have not proved to be necessary up to now. On the other hand, the desire is that more accurate diagnostic tools will be developed in the future, since the available tools are still based on probing, which is rudimentary and could be replaced by a more precise device.

Furthermore considering the complexity of peridontal disease, it is not possible to think of the diagnosis of periodontal diseases outside the concept of integral care, which includes additional tests (glycated hemoglobin data in diabetic individuals, or the aid of other blood tests in systemically compromised individuals), but not only that, the professional during the clinical examination should remember that to take care of a human being, it is necessary to consider aspects such as: life history, family dynamics, exposure to risk factors, social aspects and psychology.

\section{Goals for Latin America - concluding remarks}

This section reviewed the important aspects of periodontal diagnosis, in an evidence-based approach, trying to summarize the state of the art, and taking into consideration the characteristics of the dental profession in Latin American countries. 
These countries have experienced continuous development in oral health care. However, the prevalence of periodontal diseases is still high and a burden of disease is observed in the region. In addition, cultural and socioeconomic characteristics are common in the area, which call for specific approaches. With the aim of increasing the quality of the profession even further, the following aspects should be considered:

a. A call for action is necessary to increase awareness of periodontal diseases to enhance the quality of oral health care and proper maintenance of teeth throughout the patient's life;

b. Dental professionals should be trained right from the undergraduate curriculum to appropriately diagnose periodontal diseases and to successfully achieve prevention of periodontal pathology;

c. Dental professionals need to increase awareness in the community about periodontal diseases. Therefore, the information that gingival bleeding is not normal, and that tooth spacing or mobility might be signs of periodontal disease, etc. should be spread. Additionally, the use of self-reported periodontal diagnosis could be utilized;

d. Dental practitioners should be aware of systemic and behavioral aspects that are linked to periodontal diseases and include them in the interview with the patient; they must be able to work at a multidisciplinary level in cases in which this is required to accomplish overall health; e. Oral health professionals should routinely perform periodontal clinical examination, according to the level of disease of the patient;

f. Additional diagnostic tests should be understood as being part of periodontal diagnosis and practitioners should know how to use them to obtain their best diagnostic yield;

g. Dental professionals should understand the periodontal health/disease process to enable them to approach it correctly, either by themselves or to refer their patients for a proper approach to treatment;

h. A learning process with continuous evaluation of the new classification system is important for the purpose of standardizing periodontal diagnosis in Latin America. In addition, it is necessary to promote constant periodontal education in the dental community.

\section{Acknowledgments}

This paper was prepared for the consensus meeting titled "Periodontal disease and its impact on general health in Latin America - Latin American Consensus", promoted by the Latin American Oral Health Association (LAOHA) and Colgate Palmolive Co. with participation of experts from the region, including representatives from Periodontal Societies of Latin America. All participants had the opportunity to review the content, and eventually make their own contributions. Consensus Report was based on this paper. The authors point out that they have no conflicts of interest.

\section{References}

1. Cardoso EM, Reis C, Manzanares-Céspedes MC. Chronic periodontitis, inflammatory cytokines, and interrelationship with other chronic diseases. Postgrad Med. 2018 Jan;130(1):98-104. https://doi.org/10.1080/00325481.2018.1396876

2. Haag DG, Peres KG, Balasubramanian M, Brennan DS. Oral conditions and health-related quality of life: a systematic review. J Dent Res. 2017 Jul;96(8):864-74. https://doi.org/10.1177/0022034517709737

3. Sanz M, Ceriello A, Buysschaert M, Chapple I, Demmer RT, Graziani F, et al. Scientific evidence on the links between periodontal diseases and diabetes: consensus report and guidelines of the joint workshop on periodontal diseases and diabetes by the International Diabetes Federation and the European Federation of Periodontology. J Clin Periodontol. 2018 Feb;45(2):138-49. https://doi.org/10.1111/icpe.12808

4. Preshaw PM. Detection and diagnosis of periodontal conditions amenable to prevention. BMC Oral Health. 2015;15(S1 Suppl 1):S5. https://doi.org/10.1186/1472-6831-15-S1-S5

5. Armitage GC. Development of a classification system for periodontal diseases and conditions. Ann Periodontol. 1999 Dec;4(1):1-6. https://doi.org/10.1902/annals.1999.4.1.1 
- Periodontal disease and its impact on general health in Latin America. Section IV: Diagnosis

6. Caton JG, Armitage G, Berglundh T, Chapple IL, Jepsen S, Kornman KS, et al. A new classification scheme for periodontal and peri-implant diseases and conditions - Introduction and key changes from the 1999 classification. J Periodontol. 2018 Jun;89 Suppl 1:S18. https://doi.org/10.1002/JPER.18-0157

7. Teles R, Moss K, Preisser JS, Genco R, Giannobile WV, Corby P, et al. Patterns of periodontal disease progression based on linear mixed models of clinical attachment loss. J Clin Periodontol. 2018 Jan;45(1):15-25. https://doi.org/10.1111/icpe.12827

8. Donos N. The periodontal pocket. Periodontol 2000. 2018 Feb;76(1):7-15. https://doi.org/10.1111/prd.12203

9. Kingman A, Susin C, Albandar JM. Effect of partial recording protocols on severity estimates of periodontal disease. J Clin Periodontol. 2008 Aug;35(8):659-67. https://doi.org/10.1111/j.1600-051X.2008.01243.x

10. Silva-Boghossian CM, Amaral CS, Maia LC, Luiz RR, Colombo AP. Manual and electronic probing of the periodontal attachment level in untreated periodontitis: a systematic review. J Dent. 2008 Aug;36(8):651-7. https://doi.org/10.1016/i.jdent.2008.04.015

11. Susin C, Kingman A, Albandar JM. Effect of partial recording protocols on estimates of prevalence of periodontal disease. J Periodontol. 2005 Feb;76(2):262-7. https://doi.org/10.1902/jop.2005.76.2.262

12. Dula K, Benic GI, Bornstein M, Dagassan-Berndt D, Filippi A, Hicklin S, et al. SADMFR Guidelines for the Use of Cone-Beam Computed Tomography/Digital Volume Tomography. Swiss Dent J. 2015;125(9):945-53

13. Tugnait A, Carmichael F. Use of radiographs in the diagnosis of periodontal disease. Dent Update. 2005 Nov;32(9):536-8, 41-2. https://doi.org/10.12968/denu.2005.32.9.536

14. Armitage GC. Learned and unlearned concepts in periodontal diagnostics: a 50-year perspective. Periodontol 2000. 2013 Jun;62(1):20-36. https://doi.org/10.1111/prd.12006 